\title{
Editorial
}

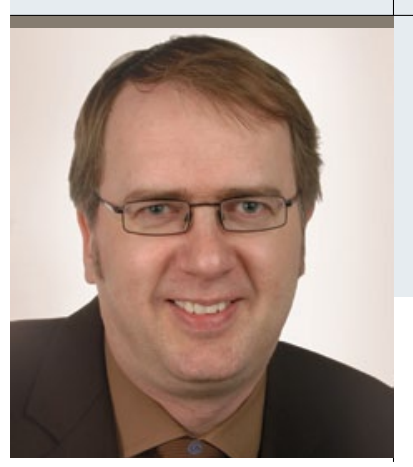

"Es wäre zu begrüßen, wenn mehr neurologische Kollegen die Weiterbildung, Spezielle Schmerztherapie' absolvieren und an der schmerztherapeutischen Versorgung teilnehmen würden."

\section{Versorgung neuropathischer Schmerzen verbessern}

B ei der Versorgung von Patienten mit neuropathischen Schmerzen liegt die Kernkompetenz in der Neurologie. In dieser Ausgabe von DNP - Der Neurologe \& Psychiater beschäftigt sich daher ein klar strukturierter CME-Beitrag mit der Pathophysiologie und der Diagnose neuropathischer Schmerzen (Strittmater \& Ostertag, Seite 36). Teil II zur Therapie erwartet Sie in der April-Ausgabe. Auch wenn der neuropathische Schmerz inzwischen regelhaft in neurologischen Fortbildungen und Übersichten vertreten ist, so ist in der Allgemeinmedizin und hausärztlichen Versorgung doch immer noch viel zu wenig zum Thema bekannt. Hier müssen wir Neurologen und Nervenärzte noch mehr Aufklärungsarbeit leisten, um zahlreiche Betroffene vor unnötigen Schmerzen zu bewahren!

Das klinische Bild neuropathischer Schmerzen kann sehr bunt sein, die Ursachen sind vielfältig. So ist zwar häufig noch bekannt, dass ein Diabetes mellitus zu Schmerzen führen kann, aber schon im groBen Gebiet der zentralen Deafferenzierungsschmerzen fehlt oft die Kenntnis über Zusammenhänge und Therapiemöglichkeiten.

\section{Schmerz oft als somatoform verkannt}

Die Bedeutung des Themas wird auch durch epidemiologische Zahlen unterstrichen. So liegt die Jahresprävalenz neuropathischer Schmerzen in Deutschland je nach Methodik der Erhebung zwischen $3 \%$ und $8 \%$; nach einem Schlaganfall klagen circa $30 \%$ aller länger Überlebenden über chronische Schmerzen, $8 \%$ klagen über einen klassischen zentralen Deafferenzierungsschmerz (früher auch Thalamusschmerz genannt). Problematisch ist die Situation auch dadurch, dass viele Betroffene nicht von Schmerzen im engeren Sinne reden, sondern den neuropathischen Schmerz mit anderen Begriffen umschreiben. Dadurch wird die Beschwerdeschilderung oft als somatoform verkannt und dann falsch diagnostiziert und behandelt. Dabei können wir die hausärztlichen Kolleginnen und Kollegen selbstbewusst darüber informieren, dass es inzwischen gute diagnostische Methoden gibt, um neuropathische Schmerzen zu identifizieren, und dass sich auch die therapeutischen Möglichkeiten enorm ausgeweitet haben.

Durch einfache Screening-Methoden wie etwa painDETECT kann das Ausmaß neuropathischer Schmerzen gut erfasst werden. Die Bandbreite der medikamentösen Möglichkeiten ist in den letzten Jahren immer größer geworden. Vor allem Adjuvantien aus den Gruppen der Antikonvulsiva und der Antidepressvia können inzwischen differenziert eingesetzt werden und haben den undifferenzierten Einsatz von peripheren Analgetika oder Opioiden abgelöst. Neuere Verfahren wie gezielte transdermale Applikationen von Substanzen oder die Neuromodulation sind in der Entwicklung, um in $\mathrm{Zu}$ kunft neuropathische Schmerzen immer stärker an der Ätiologie angelehnt behandeln zu können. Schließlich finden auch präventive Ansätze, etwa beim CRPS Typ 1 oder bei der postzosterischen Neuralgie Eingang in die Therapie, so dass nicht nur die Behandlung von chronischen neuropathischen Schmerzen, sondern auch die präventive Behandlung in der Schmerztherapie ihren Platz bekommt.

Insgesamt nehmen wir Neurologen und Nervenärzte dabei eine Vermittlerrolle ein. Zum Einen müssen wir Überzeugungsarbeit leisten, dass einfache neuropathische Schmerzen in der hausärztlichen Praxis erkannt und behandelt werden. Zum Anderen sollten wir die Kompetenz präsentieren, Patienten mit ausgeprägten neuropathischen 
Schmerzen selbst zu diagnostizieren und zu behandeln. Schließlich ist es auch unsere Aufgabe, Patienten mit chronifizierten und komplexen neuropathischen Schmerzen in die spezielle Schmerztherapie zu vermitteln, wo mit multimodalen Konzepten ebenfalls gut geholfen werden kann. Leider überlassen wir dieses Feld häufig den anästhesiologischen Kollegen, obwohl gerade bei neuropathischen Schmerzen die Kernkompetenz in unserer Disziplin liegt. Es wäre daher zu begrüßen, wenn mehr neurologische Kollegen die entsprechende Weiterbildung absolvieren und dann auch an der schmerztherapeutischen Versorgung teilnehmen würden.

\section{Eigenständige neurologische Intensivmedizin}

Die neurologische (oder auch neuromedizinische) Intensivmedizin hat seit vielen Jahren ihre Eigenständigkeit entwickelt und ausgebaut. Es hat sich sowohl in diagnostischer als auch in therapeutischer Hinsicht gezeigt, wie wichtig eine eigenständige neurologische Intensivmedizin ist. Die Arbeitstagung Neurointensiv- und Notfallmedizin (ANIM) hat dazu nicht unerheblich beigetragen. Einen Bericht von der Jahrestagung 2013 in Mannheim lesen Sie ab Seite 10.

Leider ist das Konzept der neurologischen Intensivmedizin in letzter Zeit in Gefahr geraten. Andere Disziplinen möchten unter dem vordergründigen Argument einer Rationalisierung ebenfalls neurologische Krankheitsbilder intensivmedizinisch behandeln. In diesem Zusammenhang steht auch das Vorhaben zunehmend interdisziplinäre Notaufnahmen zu etablieren, in denen die Neurologie allenfalls noch eine untergeordnete, konsiliarische Rolle übernimmt. Dadurch werden Patienten- ströme so gelenkt, dass eine neurologische Intensivmedizin entbehrlich gemacht wird. Dies ist aus wenigstens zwei Gründen riskant: So müssen intensivmedizinische neurologische Krankheitsbilder auch neurologisch betrachtet werden. Die systemische Beurteilung von Symptomen, das Wissen um den Faktor Zeit, die perspektivische Einordnung neurologischer Ausfälle, all dies ist Kernkompetenz der Neurologie. Außerdem müssen wir daran denken, dass Intensivmedizin Teil unserer Weiterbildung ist. Es ist gut, dass während der Facharztausbildung mindestens ein halbes Jahr auf einer neurologischen Intensivstation gearbeitet werden muss. Diese Weiterbildung sollten wir nicht internistischen oder operativen Intensivstationen überlassen.

Die Deutsche Gesellschaft für Neurologie hat dieses Problem erkannt und mit mehreren Initiativen begonnen, sowohl die neurologische Intensivmedizin zu erhalten als auch die Notfallkompetenz der Neurologie stärker in den Blick zu rücken. Möge diesen Initiativen Erfolg beschieden sein, damit auch in Zukunft die Neurologie als ganzheitliches Fach ihre Schwersterkrankten und ihre Notfälle selbst behandeln wird.

Mit kollegialen Grüßen,

Ihr

Stefan Evers

Professor Dr. med. Dr. phil. Stefan Evers

Chefarzt der Neurologischen Klinik

Krankenhaus Lindenbrunn, Coppenbrügge

\section{e. Akademie - Automatische Übermittlung Ihrer CME-Punkte an die Ärztekammer}

Die in der e.Akademie erworbenen CME-Punkte können auf Ihren Wunsch hin direkt an die Ärztekammer übermittelt werden.

So einfach geht's:

- Einheitliche Fortbildungsnummer (EFN)

hinterlegen

Möchten Sie Ihre in der e.Akademie gesammelten

CME-Punkte direkt an Ihre Ärztekammer übermitteln,

hinterlegen Sie bitte Ihre EFN bei der Registrierung.

Wenn Sie bereits bei springermedizin.de registriert sind,

können Sie Ihre EFN jederzeit unter dem Punkt Meine

Daten nachtragen. Ihre CME-Punkte werden dann auto-

matisch an Ihre Ärztekammer übermittelt.
- Weitere Informationen

Weitere Informationen zur elektronischen Punkteübermittlung der Bundesärztekammer finden Sie unter www.eiv-fobi.de.

Teilnehmen und weitere Informationen unter: springermedizin.de/eAkademie

Unser Tipp! Noch mehr Fortbildung bietet das e.MedKomplettpaket. Hier stehen Ihnen in der e.Akademie alle Kurse der Fachzeitschriften von Springer Medizin zur Verfügung.

Testen Sie e.Med gratis und unverbindlich unter springermedizin.de/eMed 